\title{
Research on Grid-connected Technology of Energy Storage System with Liquid Metal Batteries
}

\author{
Dalei Wang ${ }^{1}$, Cheng $\mathrm{Xu}^{1}$, Fangfang Zhu ${ }^{1}$, Kangli Wang ${ }^{1, *}$, Kai Jiang ${ }^{2}$ \\ ${ }^{1}$ College of Electrical and Electronic Engineering, Huazhong University of Science and Technology, \\ Wuhan, 430074, China \\ ${ }^{2}$ College of Materials Science and Technologies, Huazhong University of Science and Technology, \\ Wuhan, 430074, China \\ *email: klwang@hust.edu.cn
}

Keywords: Liquid Metal Battery; Grid Connection; DC-DC Converter; Inverter

\begin{abstract}
Liquid metal batteries (LMBs) are cheap and efficient energy storage systems developed in recent years. The large scale applications of LMBs are critical to the utilization of renewable energy and the development of advanced smart grid. This study focuses on the design of grid connection based on LMBs. Considering the LMB's unique characteristics, a second-order RC model is introduced and the suitable DC-DC converter is selected as well as the inverter. The system can be connected to the power system of $380 \mathrm{~V}$ through a smoothing reactor and a shunt filter. This research establishes the foundation researches for the application of LMBs in the grid energy storage filed.
\end{abstract}

\section{Introduction}

The energy storage technology has got plenty of attention because of the energy crisis and smart grid development. Energy storage battery has advantages in its clean, efficient, convenient features, becoming one of the most popular storage technologies. However, the high cost of existing battery technologies, combined with relatively short cycle life, such as lithium-ion batteries, sodium sulfur batteries and flow batteries, create a large barrier for their applications in the large-scale energy storage market [1].

In recent years, the emergence of liquid metal battery provides a new option for the grid-level energy storage application. The positive and negative metal is liquid and the electrolyte is molten salts when LMB is running. Since its electrode and electrolyte is automatically divided into three layers according to their different densities, LMB has the inherent advantages of fast transfer speed, high efficiency, stable performance and unprecedented cycle life [2].

In this paper, we study on the LMB connecting to the power system, taking full account of its special internal status and characteristics of low voltage and high current. Via electronic devices, LMB can supply power for three-phase load, and transfer the power to the grid.

\section{Design of the Grid Connection System}

\section{A. The Structure of the Grid Connection System}

Generally, the grid inverter of power generation system has the following two categories: single-level structure with DC-AC and two-level structure with DC-DC plus DC-AC. Single-level structure needs less power electronic devices, but requires isolation with general frequency transformers. In addition, the system has a large input voltage range and is cumbersome as well as inefficient, whose inverter is also too complex to design. Two-level structure first transforms DC current output by the battery with pre-DC-DC converter, and then makes use of the post-DC-AC converter to transform DC into three-phase AC [3][4]. The two-level structure can also be divided into two kinds according to the different isolated position. Post-isolation also needs general frequency transformer to isolate, which has the same drawback as well as the single-level structure. Meanwhile, pre-isolation is a better choice as it achieves high-frequency isolation and reduces the 
transform's size and weight.

In this paper, we choose two-level structure with pre-isolation to make up the low-voltage battery system. The source voltage, either $24 \mathrm{~V}$ or $48 \mathrm{~V}$, is boosted to a certain level through DC-DC converter, and then output by the inverter. Due to the low cell voltage of LMB (voltage window is $0.5-1.2 \mathrm{~V}$ and voltage platform is generally about $0.7 \mathrm{~V}$ or $0.8 \mathrm{~V}$ ), to string to the same voltage level needs more number of batteries compared to solid-state batteries, such as lithium-ion batteries. This will bring great difficulties to the battery management system (BMS) and increase the probability of failure outage. Therefore, the lower power supply voltage, $24 \mathrm{~V}$, is more appropriate.

The ratio of high-frequency transformer used in pre-isolation should not be too high. A higher ratio will lead to poor coupling, larger leakage inductance and increased distributed capacitance, so that no-load current increases rapidly [5]. Therefore, in order to achieve the purpose that low direct voltage $24 \mathrm{~V}$ is converted to alternating voltage $380 \mathrm{~V}$, we will use the boost circuit and the isolation transformer to step up voltage, which could reduce the ratio of the isolation transformer.

\section{B. Model of Liquid Metal Battery}

In this paper, the battery module used in the simulation consists of series and parallel liquid metal battery equivalent circuit model. In order to achieve the design requirement that a battery pack reaches $24 \mathrm{~V}$ voltage platform, the battery pack consists of 30 cells in series. A specific number of battery packs in parallel can meet its current and capacity requirements.

The second-order Thevenin equivalent circuit model is applied to the liquid metal battery cell shown in Fig. 1[6]. Ideal voltage source $U_{\text {oc }}$ represents the open circuit voltage of the battery; resistance $R_{0}$ is the battery's ohmic internal resistance; $R_{p}, C_{p}$ and $R_{w}, C_{w}$ represent polarization and diffusion respectively. Through HPPC test and parameter identification, we can establish functions between parameters and LMB's state of charge(SOC), and then develop a liquid metal battery cell model in MATLAB/Simulink. Comparing the real voltage curve and the simulated one, the model's accuracy of LMB is proved.

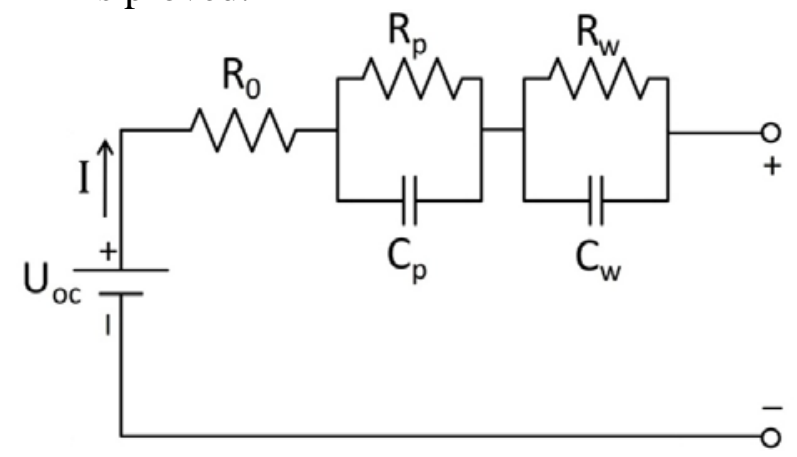

Fig.1. The second-order Thevenin equivalent circuit model

Since the consistency of batteries in series can be improved by the balanced management in BMS, it is simply thought that the model of the battery module is a superposition of 30 cell models. Through a gain of 30 , the LMB cell model is connected to the energy storage system as a battery subsystem.

Due to the high switching frequency of power devices, the sampling time need to be set shortly, usually microseconds. Since a full $0.2 \mathrm{C}$ discharge requires $5 \mathrm{~h}$, it's too long to simulate the entire energy storage system, easily causing the system crash. Therefore, in order to verify the effect of the network design, the integration time per unit of the cell model is set to $1 \mathrm{~s}$ and the system step is set to be $5 \times 10^{-6} \mathrm{~s}$. The battery can be discharged within $10 \mathrm{~s}$ at $0.2 \mathrm{C}$ rate, which greatly reduces computations.

\section{Bidirectional Current-fed Half-bridge DC-DC Converter with Active Clamp}

Bidirectional DC-DC converters are widely applied in battery storage systems. On the one hand, the excess power produced by renewable energy power plants or normal power plants can charge the battery system through the bidirectional DC-DC converter when the power system load is in the low state. On the other hand, the battery system can supply power for the grid when the power 
system load in the peak state. Meanwhile, two-way flow of energy between bidirectional DC-DC converter and the power grid can ensure the stability of the DC bus voltage [7].

The bidirectional DC-DC converter has two types, isolated and non-isolated. For this paper, we choose the former one. Isolated bidirectional DC-DC converter has different categories, including a fly-back converter, a forward converter, a push-pull converter, a full-bridge converter and a current-fed half-bridge converter [8].

Bridge circuit can be used in high power situations and has a high reliability, which is developed rapidly in the bidirectional DC-DC converter. Since the high-voltage and low-voltage side of a bidirectional full-bridge DC-DC converter has no inductors, the port current ripple will increase, which is not suitable for maintaining battery energy storage units [9]. In addition, the bidirectional full-bridge DC-DC converter is actually a buck circuit. Therefore, the current-led half-bridge bidirectional DC-DC converter is more suitable for the occasion on renewable energy power generation and grid connection.

This paper studies on the bidirectional current-fed half-bridge DC-DC converter with active clamp shown in Fig.2. Since adding coupled inductors to the low-voltage side, the power density of the converter is improved. The two series half-bridges can reduce the voltage stress on the high side [10]. By adding the active clamp circuit composed of $S_{1}, C_{d 1}$ and $S_{3}, C_{d 2}$, it can effectively clamp the main switch voltage and wipe off the voltage spikes and shocks. It also can realize zero-voltage-switching in a certain condition [11]. As both sides of the isolation transformer can be in a boost mode, the bidirectional current-fed half-bridge DC-DC converter with active clamp could make the ratio of the transformer not to be large.

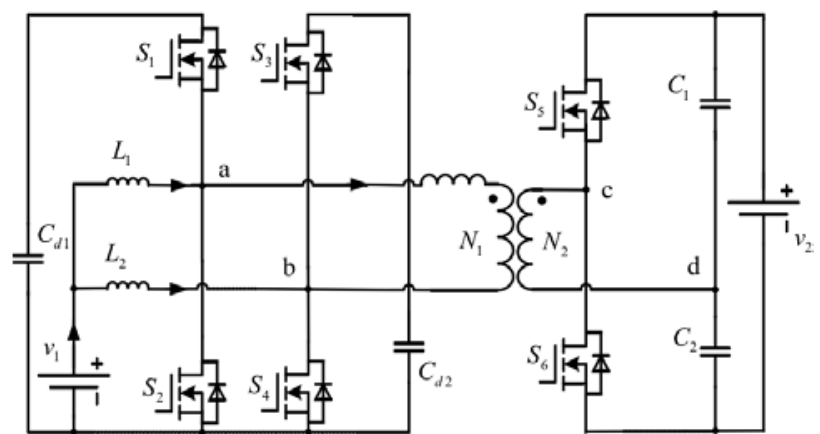

Fig.2. Bidirectional current-fed half-bridge

DC-DC converter with active clamp

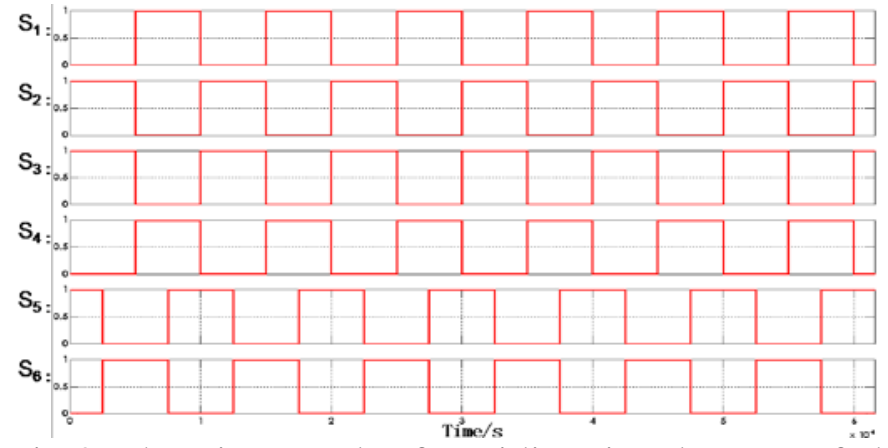

Fig.3. The trigger pulse for Bidirectional current-fed half-bridge DC-DC converter with active clamp

The output voltage of the battery in discharge is time-varying. With the input voltage in a certain range, the DC voltage output by the converter need to be nearly constant. By controlling the pulse waveform of the power switch, it can realize the transformer primary and secondary voltage match over a wide input voltage range, which reduces the circulation of the transformer, expands the scope of soft switching and achieves higher efficiency [9].

In this research, we sample the DC voltage output by the DC-DC converter and generate PWM with the difference between the feedback value and the voltage reference by PI control, limiter and logic operations. The trigger pulse waveform for the DC-DC converter is shown in Fig.3.

\section{Three-level Neutral Pointed Clamped (NPC) Inverter}

In this paper, the tree-level NPC inverter is proposed for its advantages of high switching frequency, low voltage for each switch device, and good export waves [12], which improves the system reliability. Fig. 4 shows the topology of three-level bridge inverter in the Simulink library. Its inherent NPC structure meets the requirements of this study. IGBT is used here for power devices.

The triangular carrier disposition method for the inverter modulation is as follows:

1) use the three-phase sinusoidal voltage and current output by the filter as input of the controller, and get the reference current in direct-axis by comparing the input voltage and the corresponding reference voltage in the DC side of the inverter while the quadrature-axis reference current is zero;

2) calculate direct- and quadrature-axis voltage by PID control according to direct- and 
quadrature-axis reference current and corresponding components of the three-phase sinusoidal current, and then calculate the three-phase modulation voltage;

3) use the three-phase modulation voltage as the modulation wave, and use the triangle wave as the carrier;

4) two triangle carriers which have the same amplitude and frequency distribute symmetrically among the positive and negative part of the modulation wave, and then PWM signal is generated by comparing their logical relation [13] shown in Fig.5 [14].

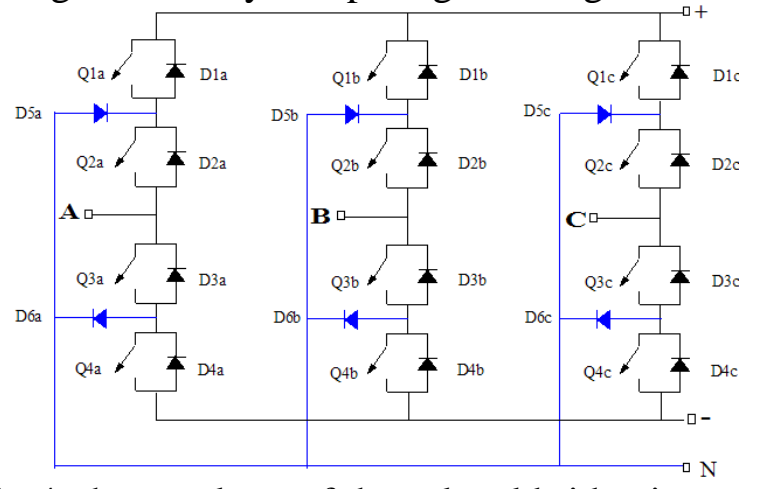

Fig.4. the topology of three-level bridge inverter

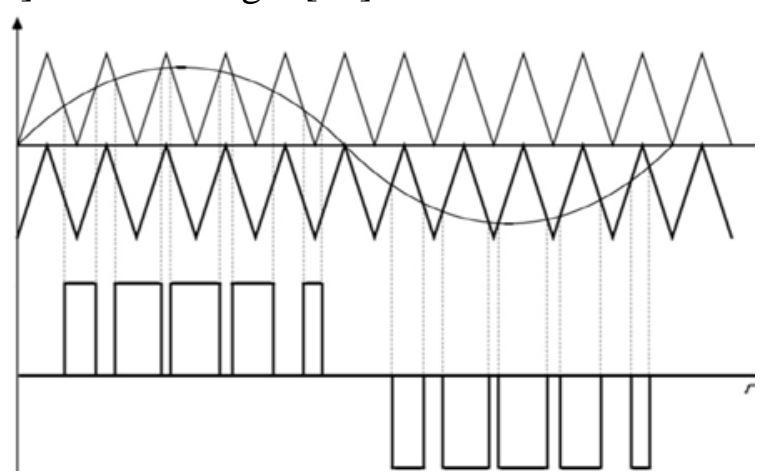

Fig.5. Schematic diagram of PWM

\section{Simulation Results of the Model}

A liquid metal battery energy storage system consists of the following components in turn: batteries, a DC-DC converter, an inverter, smoothing reactors, shunt filters, loads and a $380 \mathrm{~V}$ system. The model is established in Simulink and the results are analyzed.

The voltage output by the model is shown in Fig.6. The discharge time at $0.2 \mathrm{C}$ rate is shortened to 10 seconds while the voltage range is mainly between $16 \mathrm{~V}-28 \mathrm{~V}$.

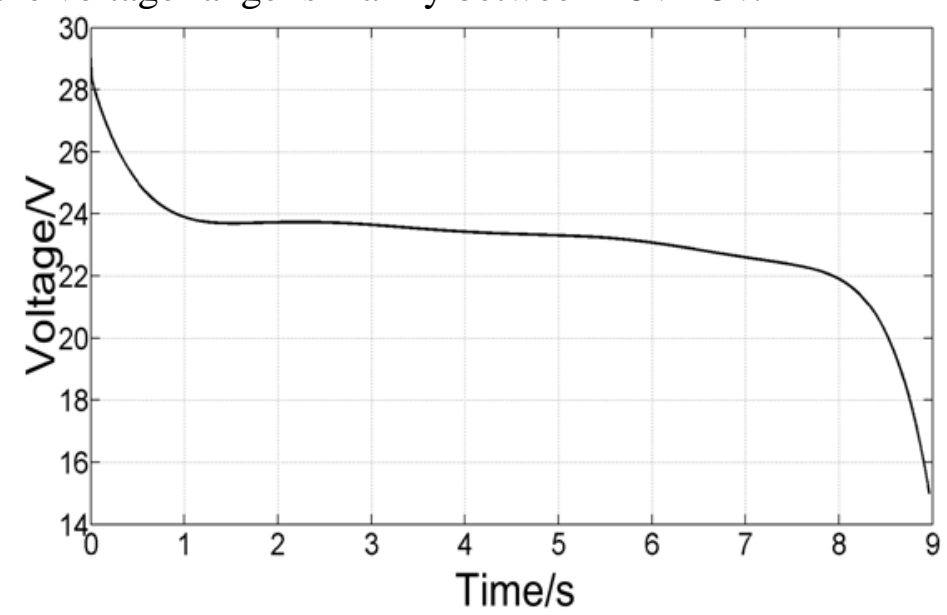

Fig.6. Output voltage of 30 liquid metal batteries in series

The RMS voltage output by the inverter is shown in Fig.7. It is proved that this design of grid connection can achieve a substantially constant output voltage under a wide range of input voltage. Through a large quantity of simulations, the inverter output RMS voltage can keep around 380V, which meets the requirement of the standard supply voltage range $( \pm 7 \%)$.

The inverter is connected to the $380 \mathrm{~V}$ power system through smoothing reactors and shunt filters. Therefore, LMBs supply a $1 \mathrm{~kW}$ load and the single-phase voltage curve of the load is shown in Fig.8, which proves that active power output is relatively stable. In order to ensure a smooth grid voltage, reactive power can be adjusted by a shunt filter according to the actual situation.

According to the results of the simulation, the LMB energy storage system has good effects on the grid connection and can supply power for the single-phase load (220V) and the three-phase load $(380 \mathrm{~V})$. 


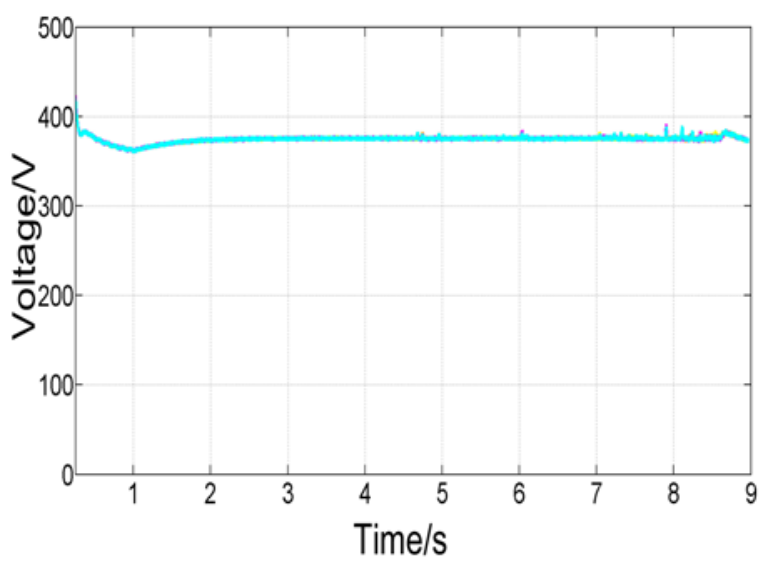

Fig.7. Output voltage (RMS) of the inverter

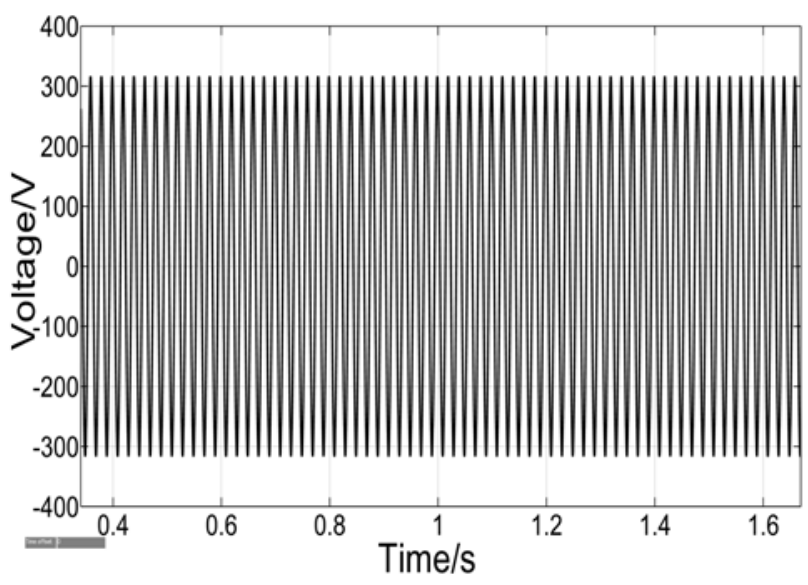

Fig.8. Voltage curve of loads (C-phase)

\section{Conclusion}

A cell model for the liquid metal battery is built in this paper. Based on the model, an energy storage system model is proposed. In order to connect the novel energy storage system to the power grid, a grid connection scheme is carefully designed. The LMB energy storage system is connected to a $380 \mathrm{~V}$ power system through a bidirectional current-fed half-bridge DC-DC converter with active clamp, a three-level neutral pointed clamped inverter, smoothing reactors and shunt filters. Through the simulation, it is proved that the design meets the requirement of grid connection and has good effects.

\section{Acknowledgement}

In this paper, the research was sponsored by the Nature Science Foundation of China (Grant 51307069) and the Innovation Fund, Huazhong University of Science and Technology (No.0118650013).

\section{References}

[1] DUNN B, KAMATH H, TARASCON J M. Electrical energy storage for the grid: a battery of choices [J]. Science, 2011,334(6058): 928-935.

[2] KIM H, BOYSEN A D, NEWHOUSE M J, et al. Liquid Metal Batteries: Past, Present, and Future [J]. Chemical Reviews, 2013,113(3): 2075-2099.

[3] Leng Guifeng. The research on inter-connection system of fuel cell distributed generation [D]. Hunan University, 2003.

[4] Bragard M, Soltau N, Thomas S, et al. The balance of renewable sources and user demands in grids: power electronics for modular battery energy storage systems [J]. IEEE Transactions on Power Electronics, 2010, 25(12): 3049-3056.

[5] Luan Song, Zhang Haifeng. Development of IGBT transformer with high power, frequency and voltage [C]. The $12^{\text {th }}$ International Conference on Electrostatic Precipitation, 2007.

[6] Lin Chengtao, Qiu Bin, Chen Quanshi. Comparison of current input equivalent circuit models of electrical vehicle battery [J]. Chinese Journal of Mechanical Engineering, 2005, 41(12): 76-81.

[7] Li Li, Liu Gang. Development of bi-directional DC-DC converter in multiple battery energy storage system [J]. Power System Protection and Control, 2011, 03: 90-94.

[8] Shoji H, Kanouda A, Saito R, et al. Isolated bidirectional DC-DC converter: U.S. Patent 7, 638, 904 [P]. 2009-12-29. 
[9] Xu Lingyu. Research on Current-Fed Bidirectional DC-DC converter [D]. Beijing Institute of Technology, 2015.

[10] Yu H, Xiang X, Zhao C, et al. Performance analysis of a ZVS bidirectional DC-DC converter with reduced voltage stress on high voltage side [C]. Applied Power Electronics Conference and Exposition (APEC), 2013 Twenty-Eighth Annual IEEE. 2013: 44-49.

[11] Kunrong Wang, Fred C.Lee, Jason Lai. Operation principle of bi-directional full-bridge DC/DC converter with unified soft-switching scheme and soft-starting capability [C]. Applied Power Electronics Conference and Exposition, 2000. APEC 2000. Fifteenth Annual IEEE.

[12] Feng Jigui. Research on PWM Control Method of Three-Level Neutral-Point-Clamped Inverter [D]. Hefei University of Technology, 2009.

[13] Liu Fengjun. Technology of Multilevel Inverter and its Application [M]. Beijing: China Machine Press, 2007.

[14] Li Yongdong, Gao Yue, Hou Xuan. Large-capacity Multi-level Converters[M]. Beijing: Science Press, 2005: 94-102. 